And now comes the point:-Is carbonic raid a poisonous gas (Dr. Christisou) ? -or does it kill by suffocation (Dr. Thomsoli)? I conceive Dr. Thomson's (?) opinion to be correct. In my own case, all the symptoms of poisoning were apparent, and none of those of suffocation, and $I$ attribute them entirely to the noxious effluvia which escaped with the carbonic acid gas; for unless the carbonic acid did really gravitate, which is not at all probable, the quantity proportioned to the atmosphere of the apartment was not sufficient, even at its maximum, to have produced suffocation; and $I$ am certain that $I$ have breathed a larger proportion of pure carbonic acid gas, without being poisoned.

In the instance of death related by $\mathrm{Dr}$. Christison, of a boy who had been teazed by a party of smiths, who held a recently-extinguished candle under his nose, the efrect never could have resulted from the carbonic acid gas, but must have been occasioned by some such noxious principle as so com. pletely enervated yours, most truly,

Charles T. Coathupe.

Wraxall, near Bristol, Oct. 26, 1838.

\section{THERAPEUTIC ACTION OF THE ERGOT OF RYE.}

\section{To the Editor of THE LANcet.}

SIR:-I trust that the few remarks I am about to make upon the action of the secale cornutum, will not be unacceptable to the generality of your readers. In the cases which $I$ published in the 24th and ist number's for Sept. 16th and Oct. 7 th, 1837, of the "Lon. Med. and Surg. Journ.," I attempted to show the specitic properties of this powerfin remedy, and my more extended experience, within the last few months, still further confirms my opinion of its utility in promoting uterine action. It has been stated by many practitioners of erninence, that the use of the ergot not only produces most alatming consequences to the mother, but likewize, in some instances, proves fatal to the child, and that it occasions retention of the placenta. Such effects I have not witnessed, as I al ways endeavour to prevent the sudden expulsion of the body of the foetus resulting from the full action of the ergot, and thus to allow the uterus to con tract gradually on the secundines, which I invariably find lying within the vagina.

Such being the grounds upon which the opinions respecting the inutility of the ergot are based, I am led to observe, in difering from these opinions, that the drug, when productive of unpleasant effects, must have been either deteriorated in quality or administered to persons of a lencophlegmatic temperament. The only symptoms which $I$ have noticed when given to such individuals have been a sensation of sinking at the pit of the stomach, together with irregularity in the beart's action. In such cases, I gese. rally administer it in a small quantity of warm ale or spirit, which prevents such an oecurrence. Ingleby, in his "Treatise on Uterine Hxmorrhage," remarks that, "Po denounce the ergot, and deny its eficacr, without an experimental acquaintance with its action, is not only most unplilosophical but altogether inconsistent with patient re. search ;" and I feel confident that if the spipit of the above quotation had been properiy observed, the number of failures would have been comparatively few, since, in m own practice, the action of the ergot has been as manifest as that of opium or mpr. cury.

My experience leads me to consider the following appearances as indicative of the genuineness of the ergot:-If the powder be put into a small vessel (a cup for instance), and about $\overline{3} \mathbf{j}$. of boiling water poured thereon, then immediately covered and allowed to stand for a fer seconds on removing the cover, the ergot should remain totally insoluble, and the infusion should assume a deep pink colour; on the contrary, if small portions of the ergot be seen floating on the surface of the water, and the infusion present a milky appearance then the action of the remedy cannot be relied upon, for $I$ have never known it, when given under such circumstances, to produce the least increase of uterine con. traction, but invariably to be followed by more or less of a feeling of sinking at the pit of the stomach, attended with irregula. rity of the heart's action, and total cessation of pain. The above fact has not, as far ts I am aware, been noticed by any writet, either on materia medica or nidwifers. Should the test I have named, as connected with the administration of the ergot, prove to hold true in other hands, as it has done in mine, I shall be fully rewarded for mp labour in endeavouring to find ont som way of judging of its purity jrior to its administration. Dr. Waller appears to think that if the pains are feeble, though regular, the ergot may produce increased action of the uterus; but that if the pains have ceased, its action is less to be de. pended upon, and it cannot be trusted wher it is found necessary to produce premature labour. Dr. Ramsbotham, in one of his lectures, published in the "Med. Gazette" has enumerated several cases wherein ho produced premature labour by means of the ergot alone, and the same result has occur red, in more than one instance, in my ow practice.

CAsE 1.-I was called upon at $11,1,4$. Sept. 26th, 1838, to attend Mrs. R, a womat of stont and rubust habit, whilst in labout of her fourth child. I ascertained, on m: arrival, from her own account, that she had been in labour for more than two wety 
On examination, I found the os uteri elongated, yet thin, resembling very much the teat of the cow ; it readily admitted the entrance of the finger, when the protrusion of the membranes, for a short distance, during each pain, was perceptible. Under such circumstances, I ordered an anodyne draught to be given immediately.

27. I was again summoned this morning, about 9, A.M.; the pains were now considerably increased, but, on examiuation, $I$ found the os uteri in much the same state; the pulse being full and strong $I$ bled her to zivi., and this procured temporary cessation from pain; I then ordered the following draught to be taken directly :- Tincture of opium, 3j.; peppermint water, $\tilde{3} \mathrm{j} . ;$ and requested that I might be cent for un the return of the pain.

28. This day, about 6, A.M., I was again summoned, and found my patient suffering from the most acute pain; her bowels had been freely opened, and the pulse was slow and weak. On introducing my finger, per vaginam, I could perceive no alteration jn the condition of the os uteri. Having tried all the means that have been recommended, or which I could myself suggest, with the view of inducing regular action, and finding them of no avail, $I$ at once determined upon the administration of the ergot, and accordingly $I$ gave Mrs. R. $\ni \mathbf{j}$. of it iu $\tilde{3}_{3 s}$ of tepid water, and in ten minutes after its use the pains assumed a more true uterine character, and, on examination, I found the membranes protruding beyond the elongated as uteri, which was also much reduced in len zth : the labour then proceeded gradually, and in the course of half an honr from the first employment of the ergot, she was delivered of a living son. I may here remark, that the mother and child are both doing well.

CASE 2.-On the 29th Sept., 1837, I was desired to attend Mrs. T., 40 years of age, and mother of several children. Her constitutional powers were much weakened from repeated abortions. On my arrival I found that she had miscarried about three weeks before of a four months' foetus, and the offensive smell on entering the room was sufficient at once to show that the secundines had not come away. She was exceedingly weak from loss of blood; pulse 140, weak and tbready; bowels regnlar; tongue coated with a brown fur; urine passed in small quantities, and high-coloured ; lower extremities nedematous; thirst urgent ; anorexia, \&c. \&c. On examination I could easily introduce the finger within the os uteri, and I found the placenta plugging up the mouth of the organ, and the placenta could be easily broken down by the finger. Seeing that no time was to be lost $I$ gave her $5 j$. of secale cornutum in $\xi_{j}$. of tepid water, and in an hour afterwards the placental mass was expelled in a complete state of decomposition. Immediate relief followed its expulsion. I need not detail each day's progress towards recovery, as it was rapid, for my patient was able to sit up and attend to her domestic duties in one month from this time.

The insertion of the foregoing remarks and cases in your valuable periodical will oblige, Sir, yours obediently,

Thos. H. WardLeworTh, Surgeon.

Rochdale, Oct. 31, 1838.

\section{PHENOMENA CONNECTED WTTH SEA-SICKNESS.}

\section{To the Editor of The LanceT.}

SIR :-I have read in THE LANCET reports of the two discussions which have taken place at the London Medical Society, on the subject of sea-sickness, and as the phenomena and physiology have not been much enterea into, may $I$ take the liberty of forwarding you a few lines upon the subject, which is one of great interest. I remain, Sir, your very obedient servant

Robert Stevens.

Keunington Common, Oct. 29, 1838.

Iu sea-sickness an individual perceives, principally by the incapacity of the muscles which maintain position, a confused knowledge of his relative position with fixed objects; but how this can produce such an affection can only be elucidated by examining certain facts connected with the affection itself, together with analogous affections, and our physical constitution.

Uuder whatever circumstances a person's knowledge of rest and position becomes confused, the sensation of sea-sickness, the identical affection, is produced. When travelling on a railroad, by observing the quick changes of perspective on the immediate side of the railroad, the affection can be taken up, ad libitum, by the eye ; whilst during intoxication, in bed, where the loss of position is only imaginary, it can be kept off by the eye being directed to any fixed object. On a rough sea, the loss of knowledge of position, with regard to fixed objects, exists palpably, and the affection lasts till some clue to it can be obtained; and this clue is, in some cases, the perception of perpendicular gravity, with the art of using the muscles to maintain position in the uncustomary motion. In a carriage, the perception of rest and position becomes confused more readily with the back to the horses, hence the affection takes place more readily so. All persons have not equally the innate geometrical faculty of observing and becoming accustomed to changes of position. Some are so devoid of it as to lose the points of the compass after taking one turning in a carriage; hence the degree of liability. The sailor who can keep the acconnt of position unconfused, on board 